\title{
SP1/TGF-ß1/SMAD2 pathway is involved in angiogenesis during osteogenesis
}

\author{
AO DING, YING-YING BIAN and ZHI-HONG ZHANG
}

\begin{abstract}
Department of Stomatology, The First Affiliated Hospital of USTC (Anhui Provincial Hospital), Division of Life Sciences and Medicine, University of Science and Technology of China, Hefei, Anhui 230001, P.R. China
\end{abstract}

Received June 20, 2019; Accepted November 26, 2019

DOI: $10.3892 / \mathrm{mmr} .2020 .10965$

\begin{abstract}
The relationship between osteoblasts and angiogenesis is vital for bone regeneration, especially mandibular and maxillary bones. Transforming growth factor $\beta 1$ (TGF- $\beta 1$ ) and vascular endothelial growth factor (VEGF) are closely related to angiogenesis; however, the regulatory mechanism between them remains unknown. The present study aimed to reveal this mechanism to provide novel insight for development of potential therapeutic opportunities. Western blotting and reverse transcription-quantitative PCR was used to assess the protein and mRNA expression levels in MC3T3-E1 preosteoblast cells and HUVECs, ELISAs were used to detect the expression levels of secreted VEGF, MTT assays were used to assess the viability of the cells, migratory ability was assessed using Transwell assays, angiogenesis assays were used to analyze the formation of blood vessels, and TGF- $\beta 1$ regulation was confirmed using a dual-luciferase reporter assay. The overexpression of specificity protein 1 (SP1) or TGF- $\beta 1$ increased VEGF expression levels and secretion, and promoted angiogenesis of co-cultured HUVECs. SP1 also promoted SMAD2 phosphorylation. These effects of SP1 were all reversed by the TGF- $\beta 1$ inhibitor. The VEGF inhibitor bevacizumab also reduced the SP1/TGF- $\beta 1 / \mathrm{SMAD} 2$ pathway-induced angiogenesis of preosteoblasts. In conclusion, it was demonstrated that SP1 promoted TGF- $\beta 1$ expression, activated the SMAD2 pathway and induced VEGF secretion, which may enhance angiogenic processes in preosteoblasts.
\end{abstract}

\section{Introduction}

The regenerative mechanisms of mandibular and maxillary bones have attracted increasing attention in recent decades.

Correspondence to: Dr Zhi-Hong Zhang, Department of Stomatology, The First Affiliated Hospital of USTC (Anhui Provincial Hospital), Division of Life Sciences and Medicine, University of Science and Technology of China, 17 Lujiang Road, Hefei, Anhui 230001, P.R. China

E-mail: zhangzhihong769@163.com

Key words: specificity protein 1 , transforming growth factor $\beta 1$, SMAD2, angiogenesis, osteogenesis
Most patients who undergo surgical resections require mandibular/maxillary bone reconstruction; however, some cases have reported spontaneous bone regeneration or accelerated bone repair $(1,2)$. Identifying the regulatory mechanisms behind this phenomenon may support the development of novel therapeutic strategies and provide improved treatment options for patients.

Bone regeneration involves highly integrated interactions between numerous types of cells and signaling pathways (3). In 1994, it was clinically observed that new blood vessels surrounding the site of injury could lead to the improved healing of fractures (4); it was considered that blood vessels served as scaffolds during bone regeneration, around which bone formation could take place, owing to the ability of blood vessels to provide oxygen, nutrients and other required mineral materials (3). In addition, they could function as routes for bone precursor cells to reach the site of injury (5). It has also been demonstrated that blood vessels could exert angiocrine functions, which permit blood vessels to produce a series of paracrine signals to coordinate multiple biological processes associated with osteogenesis, including proliferation, differentiation, stem cell behavior and tissue regeneration (6). It was therefore hypothesized that angiogenesis may serve a vital role in bone regeneration (7). Osteoblasts and preosteoblasts have important roles during bone regeneration-associated angiogenesis. They communicate with endothelial cells, most likely through secreting cytokines or other factors, and remodel the microenvironment; for example, osteoblasts were reported to regulate angiogenesis through secreting C-X-C motif chemokine ligand 9 (8); micro (mi)RNA (miR)-9 was observed to regulate angiogenesis through targeting the AMP-activated protein kinase pathway in osteoblasts (9); CCN family member 1 increased VEGF expression levels in osteoblasts, subsequently increasing angiogenesis (10). Nonetheless, the mechanisms regulating the interaction between osteoblasts and angiogenesis remain largely unknown.

Transforming growth factor $\beta 1$ (TGF- $\beta 1$ ) is a multifunctional cytokine associated with tissue remodeling processes, including bone regeneration and angiogenesis (10). A previous in vitro angiogenesis study revealed that TGF- $\beta 1$ induced the phosphorylation of SMAD2 and enhanced VEGF signaling, which is required for angiogenesis (11). Multiple upstream or downstream factors can affect angiogenesis through regulating the TGF- $\beta 1$ pathway; for example, leucine-rich 
$\alpha$-2-glycoprotein 1 promoted angiogenesis through modulating TGF- $\beta 1$ signaling (12); thrombospondin-4 expression in endothelial cells was observed to promote TGF- $\beta 1$-mediated effects on angiogenesis (13). TGF- $\beta 1$ is also associated with osteogenesis. It promoted osteo-induction through the PI3K/AKT/mTOR signaling pathway and synergistically functioned with bone morphogenetic protein 2 to promote the initiation and progression of osteogenesis $(14,15)$. Thus, because osteogenesis and angiogenesis are both vital processes required for bone regeneration, the TGF- $\beta 1 / \mathrm{SMAD}$ pathway may contribute to mandibular and maxillary bone repair and regeneration through promoting both osteogenesis and angiogenesis.

Specificity protein 1 (SP1) is a transcription factor involved in numerous cellular processes, such as cell differentiation and proliferation; it can directly interact with DNA and enhance gene transcription (16). SP1 was also observed to interact with SMAD and enhance TGF- $\beta 1$ signaling to promote cartilage repair in chondrocyte proliferation (17). Furthermore, the downregulation of SP1 by miRNAs, such as miR-29c and miR-223 inhibited TGF- $\beta 1$ signaling in lung cancer and gastric carcinoma $(18,19)$. SP1 also serves important roles in osteogenesis and angiogenesis; SP1 regulates human osteoblast differentiation and mineralization (20), and it is involved in the regulation of bone metabolism through the frizzled-1 precursor and peroxisome proliferator-activated receptor signaling pathways (21). In osteosarcoma cells, the downregulation of SP1 inhibited osteoblast differentiation (22), and in terms of angiogenesis, it was reported that SP1 functioned through the VEGF and epidermal growth factor receptor/p38 signaling pathways to promote angiogenesis in ovarian and pancreatic cancers $(23,24)$. Thus, it was hypothesized that SP1 could also promote bone regeneration through promoting angiogenesis and osteogenesis in mandibular and maxillary bones.

The present study aimed to reveal the regulatory mechanisms of mandibular and maxillary bone regeneration. The MC3T3-E1 cell line is a mouse embryonic osteoblast precursor cell line that is widely used to study osteoblast differentiation $(25,26)$. Although the cell line does not consist of preosteoblasts of mandibular or maxillary bones, it was used in the present study due to its differentiating potential. It was revealed that the overexpression of SP1 increased TGF- $\beta 1$ expression levels, activated the TGF- $\beta 1 /$ SMAD2 signaling pathway and promoted VEGF secretion, which facilitated the angiogenesis of preosteoblasts. These findings provided an improved understanding of mandibular and maxillary bone regeneration, and may support future studies aimed at developing novel therapeutic strategies for patients that undergo mandibular and maxillary bone resection.

\section{Materials and methods}

Cell culture and reagents. All cells were cultured at $37^{\circ} \mathrm{C}$ in a humidified atmosphere containing 5\% $\mathrm{CO}_{2}$. MC3T3-E1 preosteoblast cells were purchased from American Type Culture Collection and cultured in $\alpha$-minimum essential medium supplemented with ribonucleotides and deoxyribonucleosides (12571063; Gibco; Thermo Fisher Scientific, Inc.), 2 mM L-glutamine (Gibco; Thermo Fisher Scientific, Inc.) and $1 \mathrm{mM}$ sodium pyruvate (Gibco; Thermo Fisher Scientific, Inc.) and $10 \%$ FBS, but without ascorbic acid. HUVECs were purchased from the Shanghai Institutes for Biological Sciences, Chinese Academy of Sciences, and were cultured in DMEM (Gibco; Thermo Fisher Scientific, Inc.), supplemented with 10\% FBS (Gibco; Thermo Fisher Scientific, Inc.), $100 \mathrm{U} / \mathrm{ml}$ penicillin (Gibco; Thermo Fisher Scientific, Inc.) and $100 \mathrm{mg} / \mathrm{ml}$ streptomycin (Gibco; Thermo Fisher Scientific, Inc.). For TGF- $\beta 1$ treatment, cells were treated with 5 ng/ml TGF- $\beta 1$ (Gibco PHG9214; Thermo Fisher Scientific, Inc.) for $3 \mathrm{~h}$ directly or following pretreatment with $5 \mu \mathrm{M}$ TGF- $\beta 1$ inhibitor SB431542 (Selleck Chemicals) for $30 \mathrm{~min}$. Control groups received vehicle treatment. Bevacizumab, an anti-VEGF humanized antibody, was provided by Roche Diagnostics and $10 \mu \mathrm{g} / \mathrm{ml}$ was used to treat cells.

Cell transfection. Overexpression vector pcDNA3.1-SP1 (p-SP1) and its negative control (NC; pcDNA3.1-NC), and small interfering RNA (siRNA) of SP1 (si-SP1) and its negative control, si-NC, were transfected into cells using Lipofectamine ${ }^{\circledR} 2000$ reagent (Invitrogen; Thermo Fisher Scientific, Inc.) according to the manufacturer's protocol. For each transfection, $0.4 \mu \mathrm{g}$ of plasmid and $100 \mathrm{nM}$ of siRNA were used. Cells were cultured continuously for $4 \mathrm{~h}$ at $37^{\circ} \mathrm{C}$ and the medium containing Lipofectamine ${ }^{\circledR} 2000$ was then replaced by fresh medium. Subsequent experiments were carried out $24 \mathrm{~h}$ post-transfection unless otherwise indicated. The following siRNAs were synthesized by Sangon Biotech Co., Ltd:: si-SP1, sense 5'-AACCCACTAACACTCGGTCTA CTTCACGAGCAGCTCTGGGCTGCAGAGCC-3', antisense 5'-TGAAGTAGACCGAGTGTTAGTGGGTTCGTC GCCCAGGGACAGGAAACAC-3'; si-NC, sense 5'-ACGCGU AACGCGGGAAUUUdTdT-3', antisense 5'-AAAUUCCCG CGUUACGCGUdTdT-3'.

Co-culture of MC3T3-E1 and HUVECs. For the indirect co-culture, HUVECs and MC3T3-E1 cells were indirectly co-cultured in the same well, but separated using a $0.4 \mu \mathrm{m}$ filter insert (12 $\mathrm{mm}$ in diameter; Merck KGaA). In this co-culture system, each well had two chambers, consisting of an outer chamber (24-multiwell plate) and an inner Millicell-CM chamber. MC3T3-E1 cells $\left(1 \times 10^{4}\right)$ were seeded in the outer chamber in medium $(0.5 \mathrm{ml})$. Upon MC3T3-E1 cells reaching $50 \%$ confluence, MC3T3-E1 was transfected or treated as indicated in the figures, and in the inner chamber, a total of $2 \times 10^{4} \mathrm{HUVECs} /$ well were seeded in $0.5 \mathrm{ml}$ medium and the inserts containing HUVECs were placed into the wells of MC3T3-E1 cells. In control cultures, the cell inserts without MC3T3-E1 were placed in the control wells.

Reverse transcription-quantitative PCR (RT-qPCR). Total RNA was extracted from cells $\left(5 \times 10^{6}\right)$ using TRIzol ${ }^{\circledR}$ reagent (Invitrogen; Thermo Fisher Scientific, Inc.), being treated with $1 \mathrm{ml}$ TRIzol according to the manufacturer's protocol. A total of $1 \mu \mathrm{g}$ RNA was reverse transcribed into cDNA using the PrimeScript ${ }^{\mathrm{TM}}$ RT reagent kit (cat. no. RR037A; Takara Biotechnology Co. Ltd.) according to the manufacturer's protocol. qPCR was subsequently performed using a 96-well plate ABI Prism 7500 sequence detection system (Applied Biosystems; Thermo Fisher Scientific, Inc.) and SYBR ${ }^{\circledR}$ 
Premix Ex Taq II master mix (cat. no. RR820A; Takara Bio, Inc.), according to the manufacturer's protocol. The following primer pairs (Sangon Biotech Co., Ltd.) were used for the qPCR: SP1: Forward 5'-TGGGTACTTCAGGGATCCAG-3', reverse 5'-TGAGGCTCTTCCCTCACTGT-3'; TGF- $\beta 1$ : forward 5'-AGCCCGAAGCGGACTACTAT-3', reverse 5'-TCC ACATGTTGCTCCACACT-3'. The following thermocycling conditions were used: $94^{\circ} \mathrm{C}$ for $60 \mathrm{sec}$ and 40 cycles of $94^{\circ} \mathrm{C}$ for $5 \mathrm{sec}, 60^{\circ} \mathrm{C}$ for $34 \mathrm{sec}$, and $72^{\circ} \mathrm{C}$ for $30 \mathrm{sec}$. mRNA expression levels were quantified using the $2^{-\Delta \Delta C q}$ method (27), and the expression levels of were normalized to $\beta$-actin (forward 5'-CTCCATCCTGGCCTCGCTGT-3', reverse 5'-GCTGTC ACCTTCACCGTTCC-3').

Western blotting. Total protein was extracted from cells $\left(10^{7}\right)$ using $1 \mathrm{ml}$ RIPA buffer (Sigma-Aldrich; Merck KGaA) and quantified using a bicinchoninic acid assay kit (Thermo Fisher Scientific, Inc.), according to the manufacturers' protocol. A total of $30 \mu \mathrm{g}$ protein was separated by $10 \%$ SDS-PAGE. The separated proteins were transferred onto a nitrocellulose membrane and subsequently blocked for $1 \mathrm{~h}$ at room temperature with TBS-Tween 20 (TBST; $20 \mathrm{mM}$ Tris, $137 \mathrm{mM} \mathrm{NaCl}$, $0.1 \%$ Tween-20, pH 8.0) containing 5\% BSA (Sangon Biotech Co., Ltd.). The membranes were incubated with the following primary antibodies (all 1:1,000; Cell Signaling Technology, Inc.) overnight at $4^{\circ} \mathrm{C}$ : anti-SP1(5931), anti-VEGF (2463), anti-SMAD2 (5339), anti-phosphorylated (p)-SMAD2 (18338), anti-TGF- $\beta 1$ (3711) and anti-GAPDH (5174). Membranes were washed 3 times ( 7 min each) with TBST, and incubated with 1:5,000 diluted horseradish peroxidase-conjugated secondary antibodies (anti-mouse: G-21040 and anti-rabbit: G-21234, both from Thermo Fisher Scientific, Inc.) for $1 \mathrm{~h}$ at room temperature. Protein bands were visualized using the Enhanced Chemiluminescence Western Blotting Detection system (GE HealthCare Bio-Sciences), quantified using ImageJ software (version 1.47, National Institutes of Health) and normalized to GAPDH expression levels.

ELISA. Cells were analyzed for VEGF expression using the VEGF Human ELISA kit (cat. no. KHG0111; Thermo Fisher Scientific, Inc.), according to the manufacturer's instructions. Briefly, samples were mixed with diluent buffer supplied by the kit in a ratio of $1: 1$ and $100 \mu$ l was used to in each well of the 96 -well plate overnight at $4^{\circ} \mathrm{C}$. Following incubation, the plates were rinsed and incubated with biotin conjugate at room temperature for $1 \mathrm{~h}$. Streptavidin-HRP (100 $\mu \mathrm{l}$; supplied by kit) was then incubated in the plate at room temperature in dark. Plates were subsequently rinsed three times with wash buffer and incubated with $100 \mu \mathrm{l}$ of stabilized chromogen (supplied by the kit) for $30 \mathrm{~min}$ at room temperature in the dark. The reaction was stopped by adding $100 \mu \mathrm{l}$ stop solution to each well and the optical density was determined using an Epoch-256600 plate reader at a wavelength of $450 \mathrm{~nm}$ (BioTek Instruments, Inc.).

MTT assay. An MTT assay was used to analyze cell viability. Cells $(2,000$ cells/well) were cultured in 96-well plates with $100 \mu \mathrm{l}$ of growth medium. Following the various treatments, cells were centrifuged $\left(300 \mathrm{x} \mathrm{g}, 5 \mathrm{~min}, 4^{\circ} \mathrm{C}\right)$ and the supernatants discarded. The pellet was rinsed with PBS once and
$20 \mu 15 \mathrm{mg} / \mathrm{ml}$ MTT was added to each well and incubated for $4 \mathrm{~h}$ at $37^{\circ} \mathrm{C}$. Following the incubation, the culture medium was replaced by $150 \mu \mathrm{l}$ DMSO and subsequently vibrated gently for $10 \mathrm{~min}$ to dissolve the purple formazan crystals. Cell viability was analyzed by assessing the optical density (OD) using a microplate reader at a wavelength of $490 \mathrm{~nm}$ (BioTek Instruments, Inc.). The cell viability was calculated with the following formula: Cell viability $(\%)=[\mathrm{OD} 490 \mathrm{~nm}$ of treated group)/(OD490 of control group)] x100. All experiments were performed in triplicate.

Transwell migration assay. Transwell assay equipment (the chamber) was fitted with 24-well plates to form a twocompartment system (an inner chamber and an outer well). Cells in the logarithmic growth phase were collected by centrifugation $\left(300 \times \mathrm{g}, 5 \mathrm{~min}, 4^{\circ} \mathrm{C}\right)$, rinsed with $\mathrm{PBS}$ and centrifuged again $\left(300 \mathrm{~g}, 5 \mathrm{~min}, 4^{\circ} \mathrm{C}\right)$. Cells were washed twice and resuspended in serum-free medium at a density of $1 \times 10^{6}$ cells $/ \mathrm{ml}$. A total of $100 \mu \mathrm{l}$ cell suspension was plated in the upper chambers of Transwell plates and $0.8 \mathrm{ml}$ medium supplemented with $5 \%$ fetal calf serum (Hyclone, GE Healthcare Life Sciences) was plated in the lower chambers as a chemoattractant. Following incubation at $37^{\circ} \mathrm{C}$ for 24 hours, the porous membrane was isolated, fixed with $10 \%$ methyl alcohol for $30 \mathrm{sec}$, stained with $0.1 \%$ crystal violet for $20 \mathrm{~min}$ at room temperature and held between histological slides. Migratory cells found in $\geq 20 \%$ of the filter area were counted using the bright field optics of an microscope (Leica Microsystems, Inc.) at magnification of x100.

Angiogenesis assay. An angiogenesis assay was performed to detect the angiogenic ability of HUVECs co-cultured with MC3T3-E1 cells using an in vitro Angiogenesis Assay kit (cat. no. ab204726; Abcam), according to the manufacturers' protocol. Briefly, $50 \mu 1$ thawed extracellular matrix (ECM) solution was added to each well of a pre-chilled (on ice) white 96-well sterile cell culture plate and incubated for $1 \mathrm{~h}$ at $37^{\circ} \mathrm{C}$ to allow the solution to form a gel. A total of $2 \times 10^{4}$ cells/well were plated in $100 \mu \mathrm{l}$ media onto the solidified ECM gel or control wells (no ECM gel or ECM wells with Suramin). Angiogenesis factors/regulators (such as TGF- $\beta$ inhibitor, bevacizumab and supernatants of MC3T3-E1) were subsequently added to the desired wells as indicated in each figure, and cells were incubated cells for $18 \mathrm{~h}$ in a $37^{\circ} \mathrm{C}$ incubator containing $5 \% \mathrm{CO}_{2}$. Media was removed and wells were washed with $100 \mu \mathrm{l}$ wash buffer to remove the serum. A total of $100 \mu \mathrm{l}$ staining dye working dilution (1:200) was added to each well and incubated for $30 \mathrm{~min}$ at $37^{\circ} \mathrm{C}$. Endothelial tube formation was analyzed using a Leica DMI6000B light and fluorescence microscope (Leica Microsystems, Inc.) using a green filter (magnification x100).

Dual-luciferase reporter assay. The firefly luciferase reporter plasmid (pG5 luc) and constitutively active Renilla luciferase control plasmid (pRL-Renilla) were purchased from Promega Corporation. TGF- $\beta 1$ Promoter was cloned onto the firefly luciferase reporter plasmid (pG5-TGF- $\beta 1-l u c)$. 293T cells (obtained from American Type Culture Collection) were seeded in a 24 well plate $\left(2 \times 10^{5}\right.$ cells/well) and transfected with the plasmids using Lipofectamine ${ }^{\circledR} 2000$ (Invitrogen; 
Thermo Fisher Scientific, Inc.), according to the manufacturer's protocol, at $50 \%$ confluency. Following incubation at $37^{\circ} \mathrm{C}$ for $48 \mathrm{~h}$, cells were collected and the firefly and Renilla luciferase activities were detected using a Dual-Luciferase Reporter assay system (Promega Corporation), according to the manufacturer's protocol. Briefly, cells were lysed with lysis buffer for $20 \mathrm{~min}$ at room temperature and $100 \mu \mathrm{l}$ supernatant was subsequently transferred into luminometer tubes, mixed with $20 \mu \mathrm{l}$ luciferase assay reagent and signals were detected on a GloMax20/20 luminometer (Promega Corporation). Relative luciferase intensity was normalized to the Renilla signal.

Statistical analysis. Statistical analysis was performed using GraphPad Prism 7 (GraphPad Software, Inc.) and Microsoft Excel 2003 (Microsoft Corporation); data are presented as the mean \pm SD. Statistical significance between groups was determined using a one-way ANOVA with Tukey's post hoc analysis or a Student's t-test. Experiments were repeated $\geq 3$ times and all experiments were performed in triplicate. $\mathrm{P}<0.05$ was considered to indicate a statistically significant difference.

\section{Results}

SP1 promotes angiogenesis in preosteoblasts. SP1 was overexpressed in MC3T3-E1 cells to detect its effect on angiogenesis-related processes, such as migration and proliferation. In addition, due to angiogenesis being regulated by VEGF, the function of SP1 on VEGF expression was also assessed. Successful p-SP1 transfection of MC3T3-E1 cells was determined by RT-qPCR, which demonstrated that p-SP1-transfected cells expressed significantly higher levels of SP1 compared with the p-NC-transfected cells (Fig. 1A). In addition, p-SP1-transfeced cells had significantly higher mRNA expression levels of VEGF compared with the $\mathrm{p}$-NC-transfected cells (Fig. 1A). Similar results were observed in the protein expression levels of SP1 and VEGF following transfection; the expression levels of SP1 and VEGF were significantly higher in p-SP1-transfected MC3T3-E1 cells compared with p-NC-transfected cells (Fig. 1B). The concentration of secreted VEGF was also significantly higher in p-SP1-transfected cells compared with p-NC-transfected cells (Fig. 1C). HUVECs indirectly co-cultured with p-SP1-transfected MC3T3-E1 cells demonstrated significantly higher cell viability compared with HUVECs co-cultured with p-NC-transfected MC3T3-E1 cells (Fig. 1D). A Transwell assay was used to detect the migratory ability HUVECs co-cultured with MC3T3-E1 cells. HUVECs co-cultured with p-SP1-transfected MC3T3-E1 cells had significantly higher migratory ability compared with HUVECs co-cultured with p-NC-transfected MC3T3-E1 cells (Fig. 1E). A similar trend was observed in the angiogenic ability of HUVECs co-cultured with p-SP1-transfected MC3T3-E1 cells, which was significantly higher compared with p-NC-transfected cells (Fig. 1F). These findings suggested that SP1 overexpression may enhance VEGF expression and secretion of MC3T3-E1, and promote the migration, proliferation and angiogenesis of HUVEC cells.

TGF- $\beta 1$ promotes the angiogenesis of preosteoblasts by activating SMAD2 phosphorylation. Whether TGF- $\beta 1$ could affect angiogenesis in preosteoblasts was investigated by determining whether TGF- $\beta 1$ could activate SMAD2 signaling in MC3T3-E1 cells. In MC3T3-E1 cells, TGF- $\beta 1$ treatment significantly increased the ratio of phosphorylated vs. total SMAD2 protein levels compared with the control (Fig. 2A). This effect was significantly reversed in MC3T3-E1 cells pretreated with the TGF- $\beta 1$ inhibitor SB431542. To evaluate whether TGF- $\beta 1$ could affect VEGF secretion, an ELISA assay was used. TGF- $\beta 1$ treatment significantly increased the VEGF concentration in the medium compared with the control, and such function of TGF- $\beta 1$ was significantly abolished through pretreating cells with SB431542 (Fig. 2B). TGF- $\beta 1$ treatment of HUVECs co-cultured with MC3T3-E1 cells significantly increased viability, migration and angiogenic potential of HUVECs compared with the control, whereas SB431542 pretreatment significantly inhibited such functions compared with TGF- $\beta 1$-treated cells (Fig. $2 \mathrm{C}-\mathrm{E}$ ). These findings suggested that TGF- $\beta 1$ may activate the SMAD2 signaling pathway and promote VEGF secretion of preosteoblasts, which promoted the angiogenesis of co-cultured HUVECs.

SP1 promotes TGF- $\beta 1$ expression. The regulatory network between SP1 and TGF- $\beta 1$ in preosteoblasts is unclear. RT-qPCR and western blotting analysis was used to detect the silencing efficiency of si-SP; cells transfected with si-SP1 demonstrated significantly decreased mRNA and protein expression levels of SP1 compared with si-NC-transfected cells (Fig. 3A and B), which indicated that the siRNA vector was successfully transfected. The mRNA and protein expression levels of TGF- $\beta 1$ in MC3T3-E1 cells with transfected with p-SP1 or si-SP1 were significantly increased and decreased, respectively, compared with their respective controls (Fig. 3C and D). A dual-luciferase reporter assay was used to validate the regulatory relationship between SP1 and TGF- $\beta 1$. SP1 overexpression significantly enhanced TGF- $\beta 1$ luciferase activity compared with the NC (Fig. 3E). These findings demonstrated that SP1 may directly interact with the TGF- $\beta 1$ enhancer and promote TGF- $\beta 1$ expression.

SP1 promotes angiogenesis in preosteoblasts through activating the TGF- $\beta 1 / S M A D 2$ signaling pathway. The transcription factor SP1 can regulate the expression of numerous proteins, thus it was investigated whether SP1 could promote angiogenesis exclusively through the TGF- $\beta 1 /$ SMAD2 signaling pathway. In MC3T3-E1 cells, p-SP1-transfected cells demonstrated significantly increased mRNA expression levels of TGF- $\beta 1$ compared with the control group; however, the addition of the TGF- $\beta 1$ inhibitor SB431542 significantly attenuated such effect, with expression levels remaining non-significant from the control group (Fig. 4A). Similar results were observed by western blotting; protein expression levels of TGF- $\beta 1$ were significantly increased in p-SP1-transfected cells compared with the control group, while no significant difference compared with the control was observed in p-SP1-transfected cells pretreated with SB431542 (Fig. 4B). The overexpression of SP1 also significantly increased both p-SMAD and total SMAD2 protein expression levels (and the ratio of p-SMAD vs. SMAD2) compared with the control group, whereas the cells also pretreated with SB431542 demonstrated significantly reduced expression levels of phosphorylated and total SMAD2 


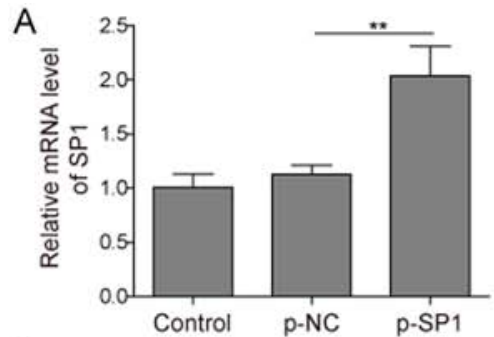

B

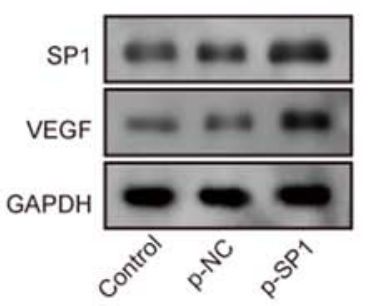

$\mathrm{E}$

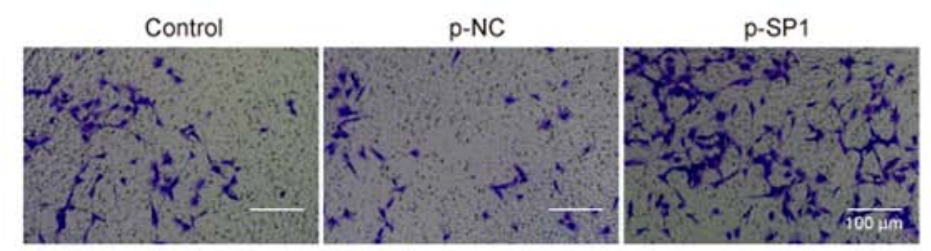

F
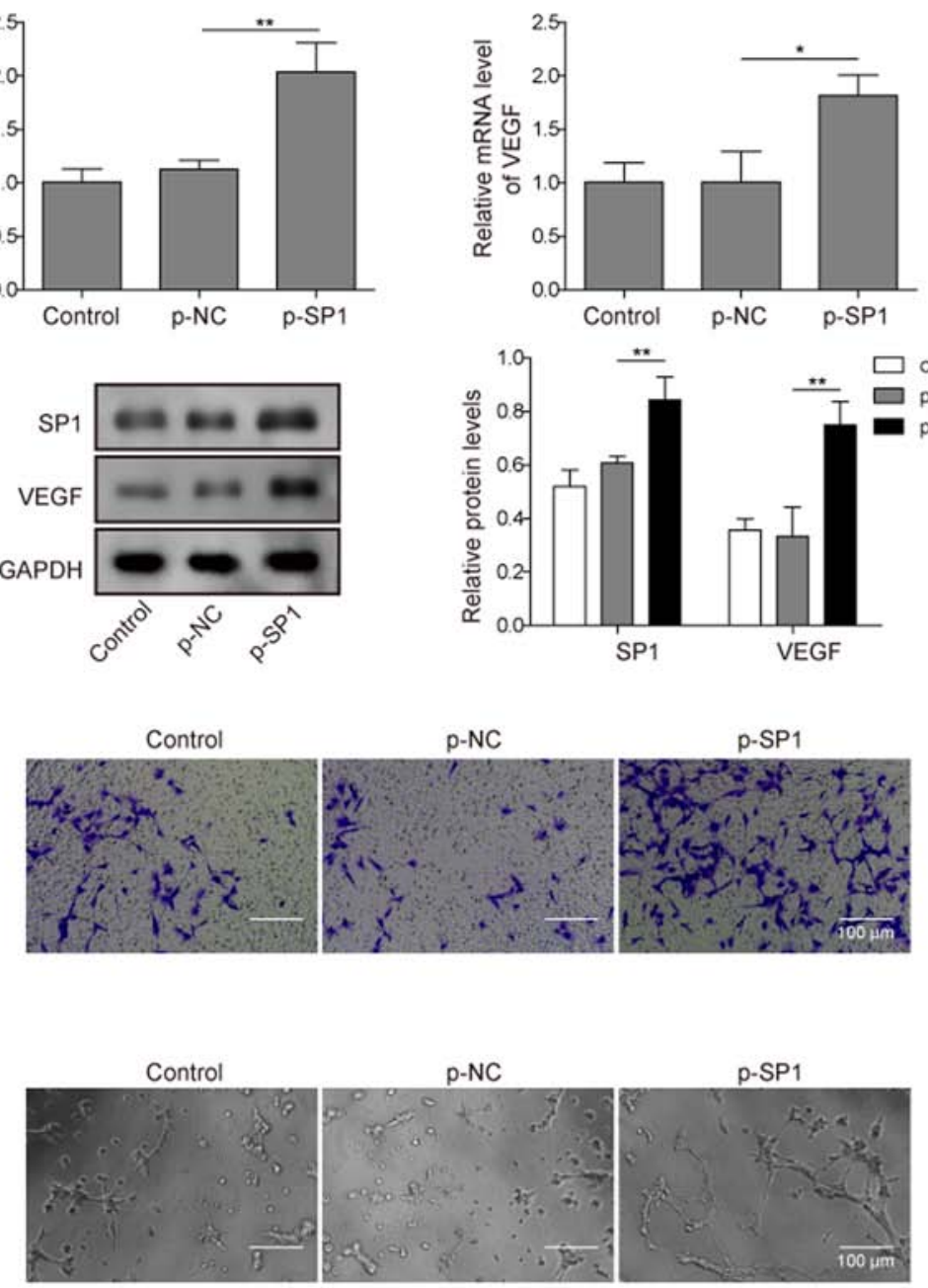

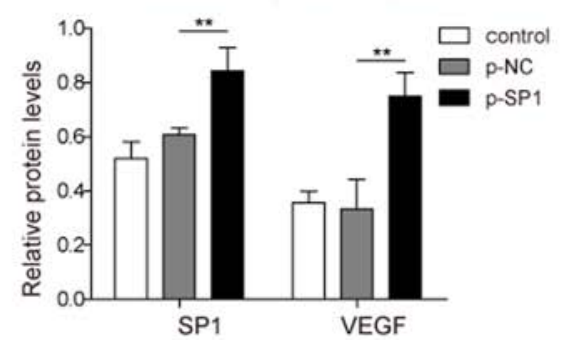

D
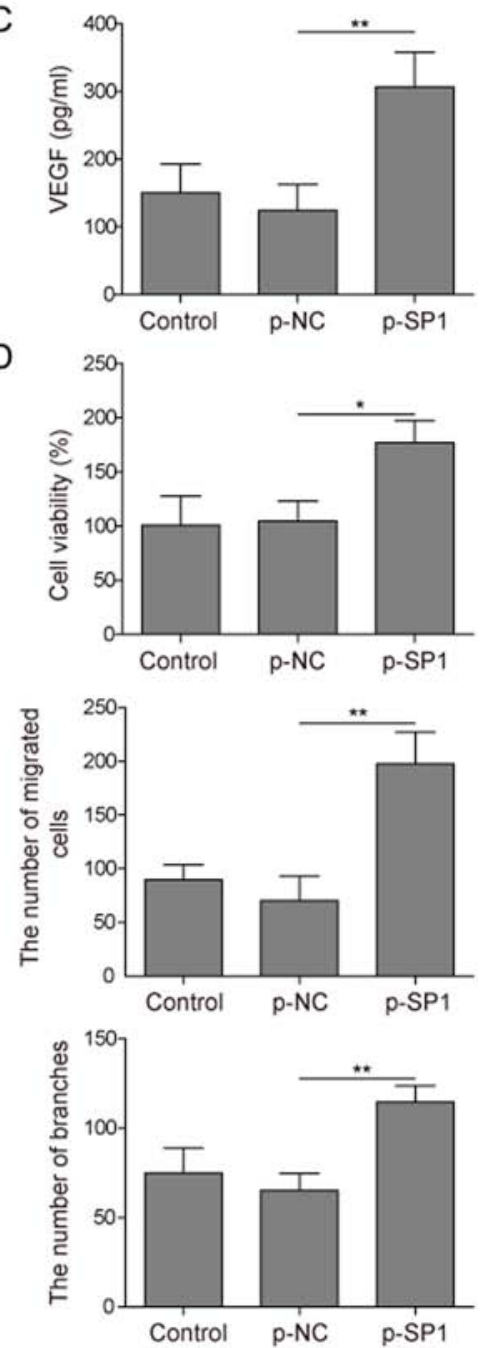

Figure 1. SP1 promotes angiogenesis in preosteoblasts. (A and B) MC3T3-E1 cells were transfected with p-SP1 or p-NC overexpression vectors and the (A) mRNA and (B) protein expression levels of SP1 and VEGF were analyzed using reverse transcription-quantitative PCR and western blotting, respectively. (C) MC3T3-E1 cells were transfected with p-SP1 or p-NC. Supernatants were collected and the protein expression levels of VEGF in the supernatants were detected using ELISA. (D-F) MC3T3-E1 cells were transfected with p-SP1 or p-NC and co-cultured with HUVECs. (D) The viability of HUVECs was detected using the MTT assay. (E) The migratory ability of HUVECs was detected using the Transwell assay. Representative micrographs are provided of HUVECs under each condition. Scale bar=100 $\mu \mathrm{m}$. (F) The capillary tube formation rate of HUVECs was detected using an angiogenesis assay. Scale bar=100 $\mu \mathrm{m}$. Representative micrographs are provided of HUVECs under each condition. Data represents one of the three independent experiments replicates with $\mathrm{n}=3$ /group for each experimental group of. Data are presented as the mean $\pm \mathrm{SD}$. ${ }^{*} \mathrm{P}<0.05$ and ${ }^{* *} \mathrm{P}<0.01$. NC, negative control; SP1, specificity protein 1 ; VEGF, vascular endothelial growth factor.

(and the ratio of p-SMAD2 vs. SMAD2) to similar levels as the control (Fig. 4C). p-SP1-transfected cells significantly increased the secretion of VEGF compared with the control, while pretreating cells with SB431542 significantly inhibited such function (Fig. 4D). HUVECs subsequently co-cultured with MC3T3-E1 cells overexpressing SP1 significantly increased the viability, migratory and angiogenic potential of HUVECs compared with the control group. These functions were all significantly inhibited by SB431542 (Fig. 4E-G). These results indicated that SP1 may promote the angiogenesis of preosteoblasts through targeting the TGF- $\beta 1 /$ SMAD2 signaling pathway.

SP1/TGF- $\beta 1 /$ SMAD2 signaling pathway promotes preosteoblast angiogenesis through regulating VEGF expression. The TGF- $\beta 1 / \mathrm{SMAD} 2$ signaling pathway regulates the expression of numerous proteins. Given that angiogenesis is stimulated by VEGF, the present study aimed to investigate whether the TGF- $\beta 1 / \mathrm{SMAD} 2$ pathway promoted the angiogenesis of preosteoblasts through VEGF. HUVECs were co-cultured with p-SP1-transfected MC3T3-E1 cells and treated with SB431542 or bevacizumab. Compared with the control group, the expression levels of secreted VEGF were increased in p-SP1-transfected and VEGF-treated cells; however, pretreatment of cells with SB431542 or bevacizumab inhibited the increased VEGF expression induced by $\mathrm{p}-\mathrm{SP} 1$ (Fig. 5A). These results indicated that the SP1 signaling pathway in MC3T3-E1 cells could promote VEGF expression in HUVECs. p-SP1-transfected and VEGF-positive cells significantly increased the cell viability compared with control group, whereas the pretreatment of cells with SB431542 or bevacizumab inhibited such function (Fig. 5B). The migratory ability of cells was detected by the Transwell assay; compared with the control group, p-SP1-transfected 

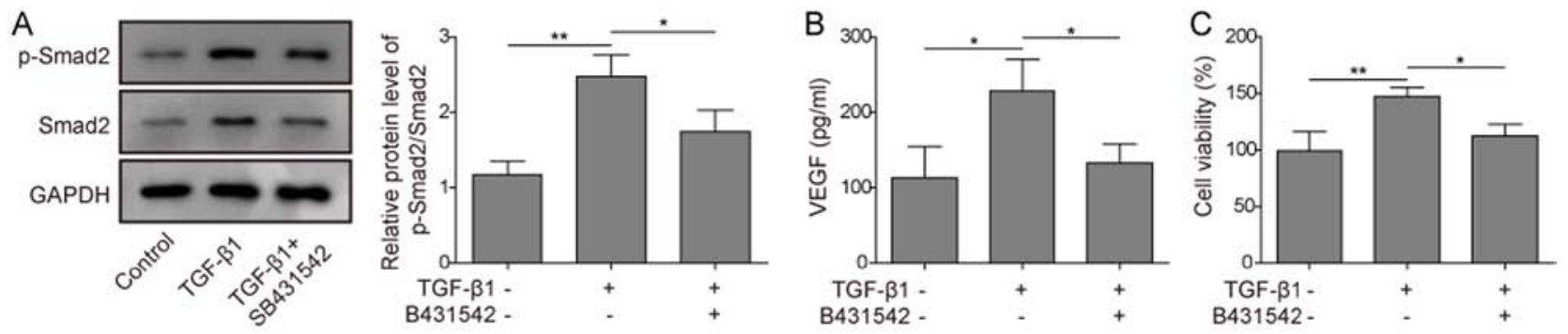

D
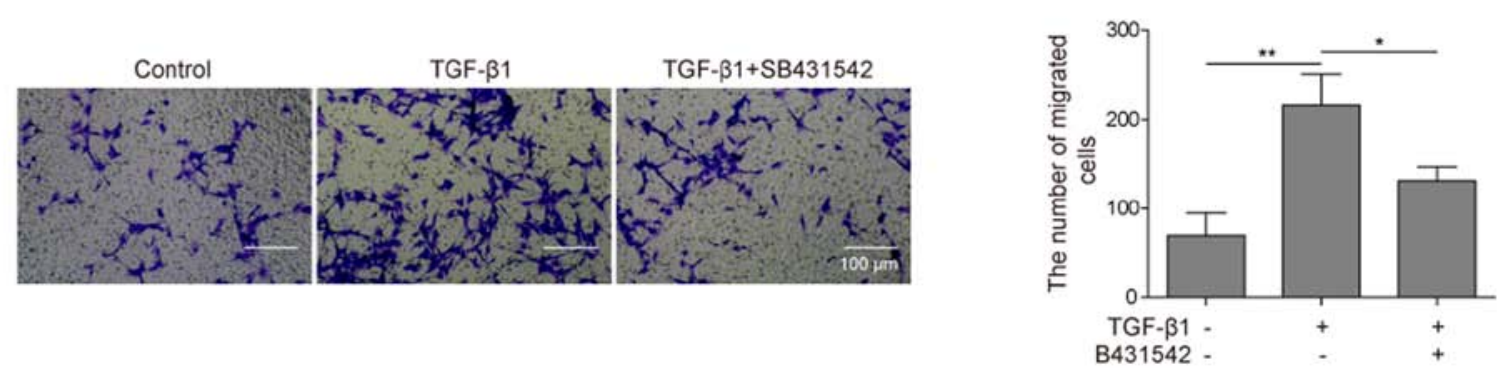

$\mathrm{E}$
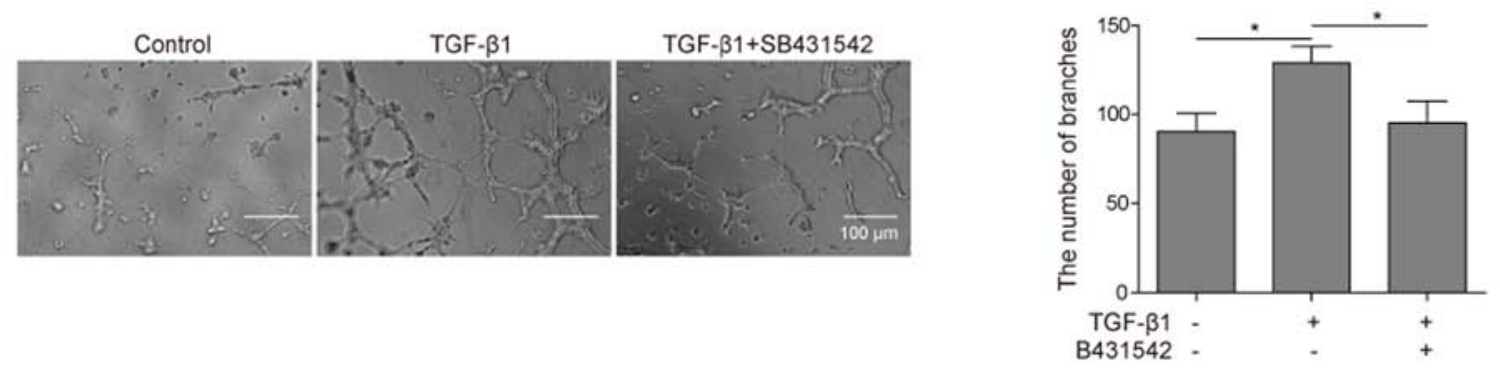

Figure 2. TGF- $\beta 1$ promotes angiogenesis in preosteoblasts through promoting SMAD2 phosphorylation. (A) MC3T3-E1 cells were treated with TGF- $\beta 1$ or TGF- $\beta 1+$ SB431542, and the protein expression levels of SMAD2 and p-SMAD2 were detected and semi-quantified using western blotting. GAPDH served as a loading control. Statistical analyses are given in the bar charts. (B) MC3T3-E1 cells were treated as described in (A) and protein expression levels of VEGF in the supernatants were detected by ELISA. (C-E) MC3T3-E1 cells were treated with TGF- $\beta 1$ or TGF- $\beta 1+$ SB 431542 and subsequently co-cultured with HUVECs. (C) Cell viability of these HUVECs was detected using the MTT assay. (D) The migratory ability of these HUVECs were detected using a Transwell assay. Representative micrographs of HUVECs under each condition are presented. Scale bar=100 $\mu \mathrm{m}$. (E) The ability of the HUVECs under each condition to undergo angiogenesis were analyzed using an angiogenesis assay. Representative micrographs of HUVECs under each condition are presented. Scale bar $=100 \mu \mathrm{m}$. Data are presented as the mean $\pm \mathrm{SD}$. ${ }^{*} \mathrm{P}<0.05$ and ${ }^{* *} \mathrm{P}<0.01$. TGF- $\beta 1$, transforming growth factor $\beta 1$; VEGF, vascular endothelial growth factor; p-SMAD2, phosphorylated-SMAD2.
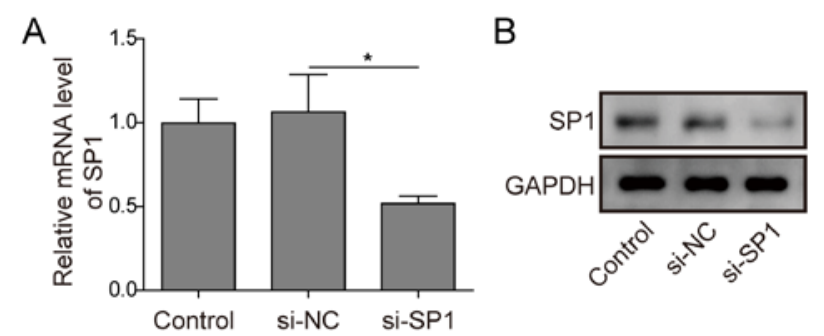

D

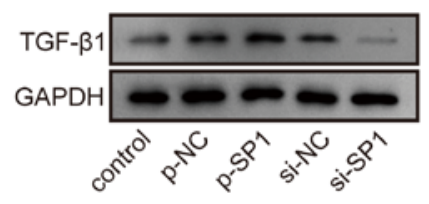

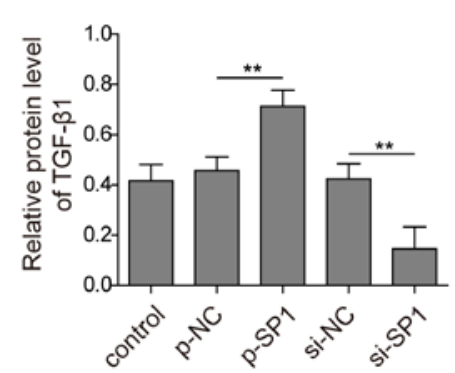
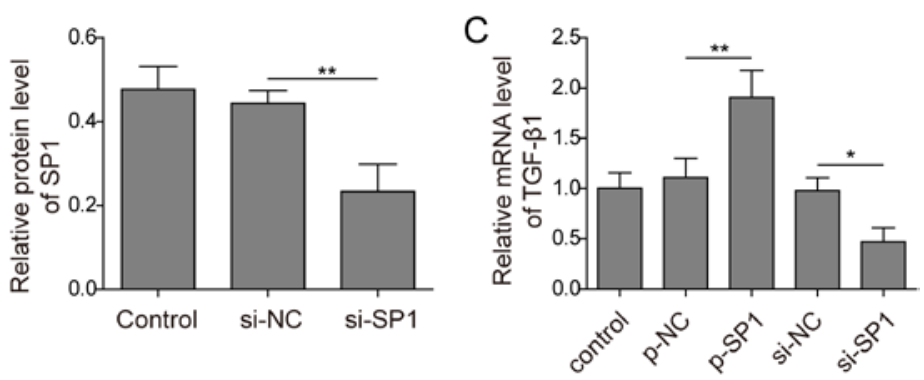

E

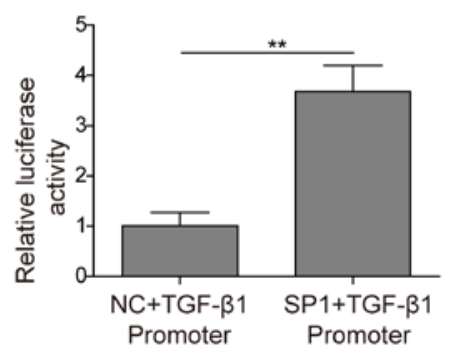

Figure 3. SP1 promotes TGF- $\beta 1$ expression. (A and B) MC3T3-E1 cells were transfected with si-NC or si-SP1 and the (A) mRNA and (B) protein expression levels of SP1 were detected by RT-qPCR and western blotting, respectively. (C and D) MC3T3-E1 cells were transfected with p-NC, p-SP1, si-NC or si-SP1 and the (C) mRNA and (D) expression levels of TGF- $\beta 1$ were detected by RT-qPCR and western blotting, respectively. (E) MC3T3-E1 cells were transfected using a dual-luciferase reporter system with a TGF- $\beta 1$ promoter and either $\mathrm{p}-\mathrm{SP} 1$ or $\mathrm{p}-\mathrm{NC}$. Data are presented as the mean $\pm \mathrm{SD}$. ${ }^{*} \mathrm{P}<0.05$ and ${ }^{* *} \mathrm{P}<0.01$. NC, negative control; RT-qPCR, reverse transcription-quantitative PCR; si, small interfering RNA; SP1, specificity protein 1 ; TGF- $\beta 1$, transforming growth factor $\beta 1$. 


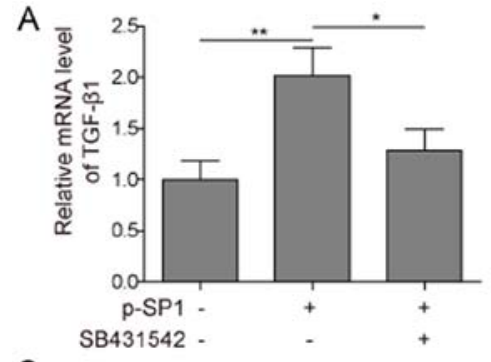

B
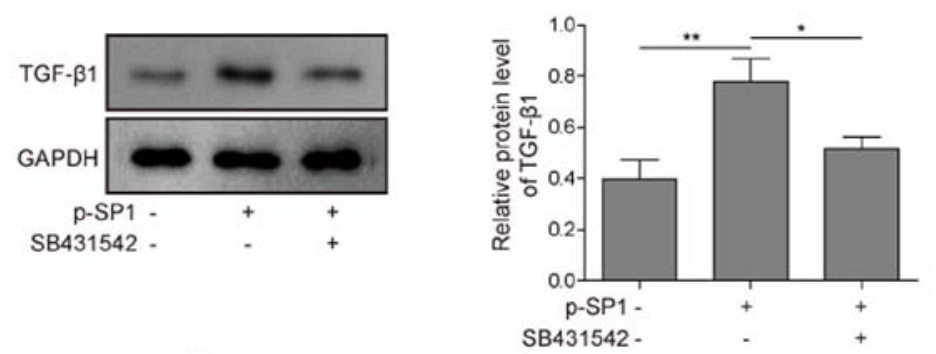

C
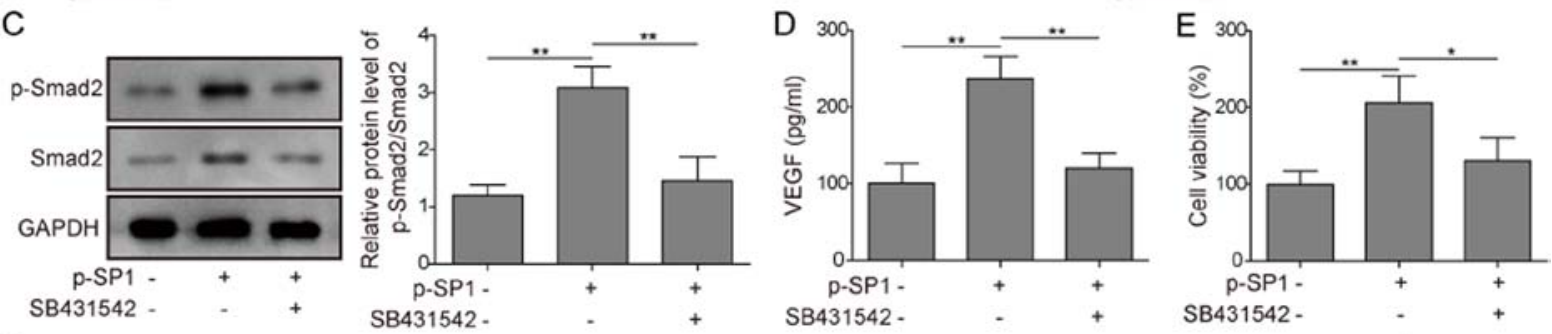

$\mathrm{F}$
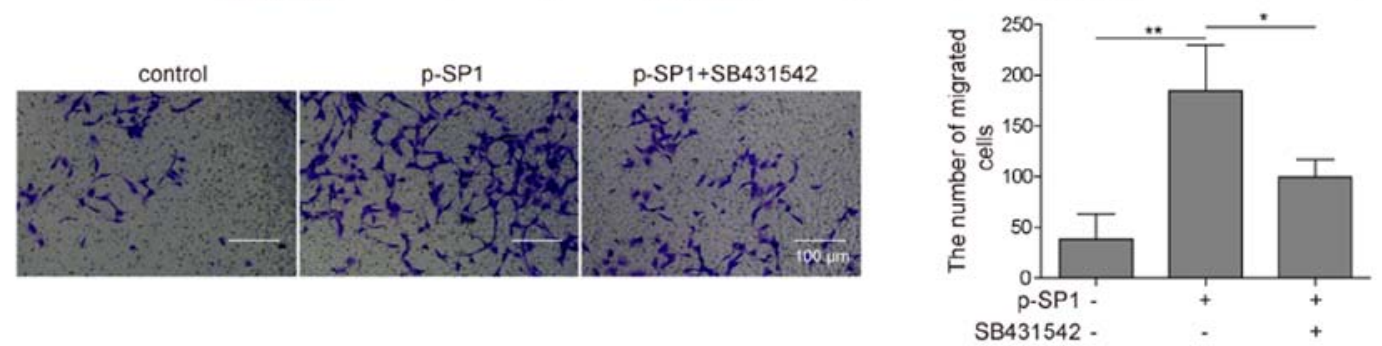

G
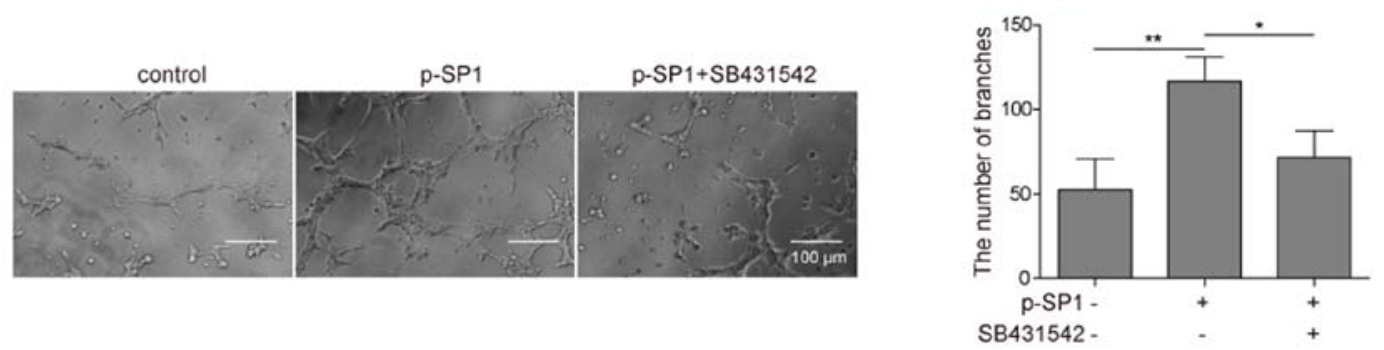

Figure 4. SP1 promotes angiogenesis in preosteoblasts through activating the TGF- $\beta 1 / \mathrm{SMAD} 2$ pathway. (A and B) MC3T3-E1 cells were transfected with p-SP1 overexpression vector and co-treated with SB431542. (A) mRNA and (B) protein expression levels of TGF- $\beta 1$ were detected by reverse transcriptionquantitative PCR and western blotting, respectively. (C) MC3T3-E1 cells were transfected with p-SP1 and co-treated with SB431542 and the protein expression levels of SMAD2 and p-SMAD2 were detected and semi-quantified using western blotting. GAPDH served as the loading control. (D) MC3T3-E1 cells were transfected with p-SP1 and co-treated with SB431542 and the VEGF concentration in supernatants were detected using ELISA. (E-G) MC3T3-E1 cells were transfected with p-SP1 and co-treated with SB431542, and co-cultured with HUVECs. (E) The cell viability of HUVECs under each condition was detected using an MTT assay. (F) Migratory or (G) angiogenic ability of HUVECs under each condition was assessed by the Transwell assay and angiogenesis assay, respectively. Representative micrographs are presented for HUVECs under each condition. Scale bar $=100 \mu \mathrm{m}$. Data are presented as the mean $\pm \mathrm{SD}$. ${ }^{\mathrm{P}}<0.05$, ${ }^{* *} \mathrm{P}<0.01$. p-SMAD2, phosphorylated SMAD2; SP1, specificity protein 1 ; TGF- $\beta 1$, transforming growth factor $\beta 1$; VEGF, vascular endothelial growth factor.

and VEGF-positive MC3T3-E1 cells significantly increased the migratory ability of HUVECs compared with the control group, whereas SB431542 or bevacizumab pretreatment significantly inhibited such function (Fig. 5C). Finally, with regards to angiogenesis, p-SP1-transfected and VEGF-positive MC3T3-E1 cells significantly increased the process of angiogenesis in HUVECs compared with the control group, whereas pretreatment with SB431542 or bevacizumab exerted similar rates of angiogenesis as the control, with no significant differences observed (Fig. 5D). These findings indicated that SP1 may promote preosteoblast angiogenesis through regulating VEGF expression.

\section{Discussion}

Angiogenesis serves an important role in bone regeneration (5); however, the regulatory mechanism is largely unknown, particularly the relationship between VEGF and TGF signaling pathways, both of which are associated with angiogenesis $(10,11)$. The present study revealed that SP1 may activate the TGF- $\beta 1 /$ SMAD2 signaling pathway and promote VEGF secretion through TGF- $\beta 1$, facilitating angiogenesis in preosteoblasts. The function of SP1 varies according to the differential genetic and expression profiles among different cell types. To the best of our knowledge, this study is the first to demonstrate that SP1 may promote angiogenesis in preosteoblasts, which is consistent with previous findings in ovarian and pancreatic cancer $(23,24)$. In these studies, SP1 mainly promoted angiogenesis through the EGFR/p38 signaling pathway; however, the present study demonstrated that SP1 promotes angiogenesis in preosteoblasts through the activation of the TGF- $\beta$ /SMAD2 signaling pathway. This difference may largely due to the variety in genetic and expressional profiles among tissue and cell types. In addition, both pathways might 
A

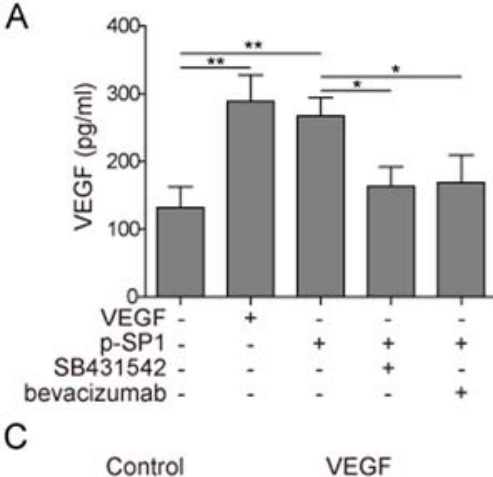

B

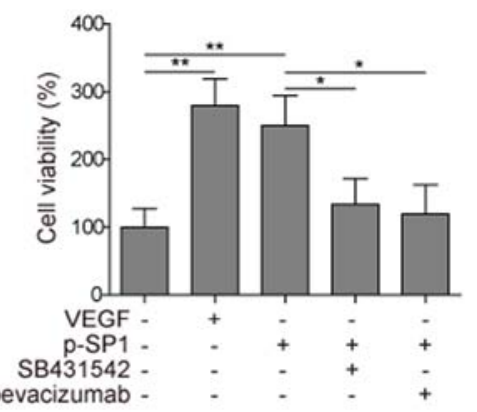

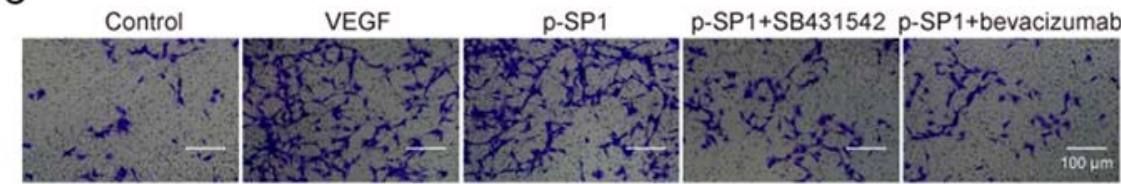

D
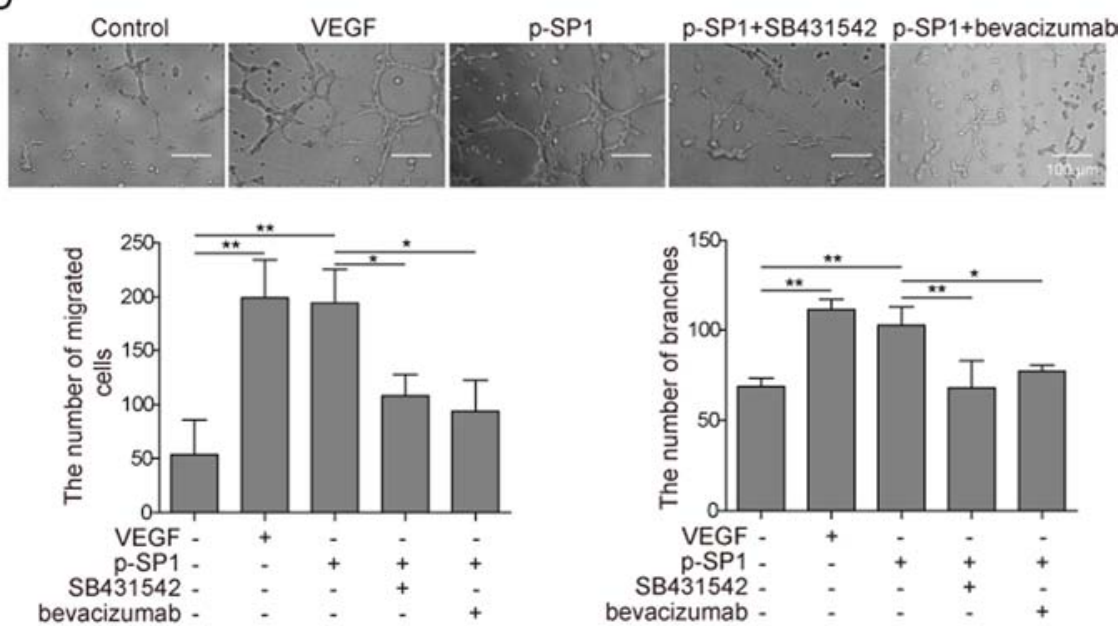

Figure 5. SP1/TGF- $31 / \mathrm{SMAD} 2$ pathway promotes preosteoblast angiogenesis through regulating VEGF expression. (A-D) MC3T3-E1 cells were transfected with p-SP1 overexpression vector and treated with VEGF, SB431542, or bevacizumab in different combinations as indicated and co-cultured with HUVECs. (A) The VEGF concentration in the supernatants was detected by ELISA 24 h post-treatment. (B) Cell viability was detected using an MTT assay. (C) Migratory ability of these cells was detected using a Transwell assay. Representative micrographs of HUVECs under each treatment condition are provided. Scale bar $=100 \mu \mathrm{m}$. (D) The ability to undergo angiogenesis was determined using an angiogenesis assay. Representative micrographs of HUVECs under each treatment condition are provided. Data are presented as the mean $\pm \mathrm{SD}$. ${ }^{*} \mathrm{P}<0.05,{ }^{* *} \mathrm{P}<0.01$. SP1, specificity protein 1 ; TGF- $\beta 1$, transforming growth factor $\beta 1$; VEGF, vascular endothelial growth factor.

contribute to angiogenesis. This result also extended our understanding of the regulation mechanism of angiogenesis.

SP1 may directly target and regulate TGF- $\beta 1$ in preosteoblasts; the association between SP1 and TGF- $\beta 1$ in the present study agreed with previous reports in other cell types, such as chondrocytes, lung cancer cells and gastric carcinoma cells $(17,18)$, where SP1 genetic knockdown affected TGF- $\beta 1$ expression. However, whether there was a direct relationship between SP1 and TGF- $\beta 1$ signaling was not explored. To the best of our knowledge, the present study is the first confirmatory study of the direct regulatory effect of SP1 on TGF- $\beta 1$ in preosteoblasts, as demonstrated by the dual-luciferase reporter assay. Through the TGF- $\beta 1$ signaling pathway, SP1 promoted the secretion of VEGF and increased levels of VEGF in the supernatant could promote HUVECs to undergo angiogenesis. This notion is supported by previous studies in multiple other cell types that the signals of the VEGF pathway were closely associated with angiogenesis, migration and proliferation $(11,28)$. In the current study, it was revealed that VEGF is under the regulation of the TGF- $\beta 1 /$ SMAD signaling pathway in preosteoblasts, which is similar to findings reported in osteoblasts $(11,14)$. Upon considering previous studies citing association of VEGF and TGF- $\beta 1$ with angiogenesis in other cell types $(7,13)$, it was concluded from this study that SP1 may directly promote TGF- $\beta 1$ expression, activate the SMAD2 pathway and promote VEGF expression and secretion, with secreted VEGF directly promoting the angiogenesis of preosteoblasts. It was also noted that although SB431542 should not affect the mRNA level of TGF-b1, under the combined effect of SP1 overexpression vector and SB431542, the mRNA level of TGF-b1 might be decreased. This could be a feed-back regulation of the influence of osteoblast angiogenesis and other changes in the microenvironment.

In conclusion, the present study revealed a direct regulatory relationship between SP1 and TGF- $\beta 1$, and uncovered its effect on angiogenesis in preosteoblasts. SP1 may stimulate the TGF- $\beta 1 /$ SMAD2 signaling pathway to promote VEGF secretion and facilitate angiogenesis. These effects are closely related with bone regeneration and repair. Thus, the results from this study may provide a theoretical basis for 
understanding the regulatory mechanisms of angiogenesis in preosteoblast cells and bone repair, and provide support for the development of novel treatment options for bone regeneration and repair in the future.

\section{Acknowledgements}

Not applicable.

\section{Funding}

This study was supported by The Anhui Provincial Natural Science Foundation (grant no. 1608085MH237).

\section{Availability of data and materials}

All data generated or analyzed during the present study are included in this published article.

\section{Authors' contributions}

AD and ZHZ designed the study. AD and YYB acquired and interpreted the data. AD prepared the manuscript and supervised the study.

\section{Ethics approval and consent to participate}

Not applicable.

\section{Patient consent for publication}

Not applicable.

\section{Competing interests}

The authors declare that they have no competing interests.

\section{References}

1. Okoturo E, Ogunbanjo OV and Arotiba GT: Spontaneous regeneration of the mandible: An institutional audit of regenerated bone and osteocompetent periosteum. J Oral Maxillofac Surg 74 1660-1667, 2016.

2. Gornitsky J, Azzi AJ and Cugno S: Spontaneous osteogenesis of a traumatic mandibular defect in the pediatric population J Craniofac Surg 30: 1999-2000, 2019.

3. Grosso A, Burger MG, Lunger A, Schaefer DJ, Banfi A and Di Maggio N: It takes two to tango: Coupling of angiogenesis and osteogenesis for bone regeneration. Front Bioeng Biotechnol 5: 68, 2017.

4. Dickson K, Katzman S, Delgado E and Contreras D: Delayed unions and nonunions of open tibial fractures. Correlation with arteriography results. Clin Orthop Relat Res 302: 189-193, 1994.

5. Hankenson KD, Dishowitz M, Gray C and Schenker M: Angiogenesis in bone regeneration. Injury 42: 556-561, 2011.

6. Ramasamy SK, Kusumbe AP, Itkin T, Gur-Cohen S, Lapidot T and Adams RH: Regulation of hematopoiesis and osteogenesis by blood vessel-derived signals. Annu Rev Cell Dev Biol 32: 649-675, 2016

7. Frohlich LF: Micrornas at the interface between osteogenesis and angiogenesis as targets for bone regeneration. Cells 8: E121, 2019.

8. Huang B, Wang W, Li Q, Wang Z, Yan B, Zhang Z, Wang L, Huang M, Jia C, Lu J, et al: Osteoblasts secrete $\mathrm{Cxcl} 9$ to regulate angiogenesis in bone. Nat Commun 7: 13885, 2016.
9. Qu J ,Lu D, Guo H, Miao W, Wu G and Zhou M: MicroRNA-9 regulates osteoblast differentiation and angiogenesis via the AMPK signaling pathway. Mol Cell Biochem 411: 23-33, 2016.

10. Chen CY, Su CM, Hsu CJ, Huang CC, Wang SW, Liu SC, Chen WC, Fuh LJ and Tang CH: CCN1 promotes VEGF production in osteoblasts and induces endothelial progenitor cell angiogenesis by inhibiting miR-126 expression in rheumatoid arthritis. J Bone Miner Res 32: 34-45, 2017.

11. Jarad M, Kuczynski EA, Morrison J, Viloria-Petit AM and Coomber BL: Release of endothelial cell associated VEGFR2 during TGF- $\beta$ modulated angiogenesis in vitro. BMC Cell Biol 18: 10, 2017.

12. Wang X, Abraham S, McKenzie JAG, Jeffs N, Swire M, Tripathi VB, Luhmann UFO, Lange CAK, Zhai Z, Arthur HM, et al: LRG1 promotes angiogenesis by modulating endothelial TGF- $\beta$ signalling. Nature 499: 306-311, 2013.

13. Muppala S, Xiao R, Krukovets I, Verbovetsky D, Yendamuri R, Habib N, Raman P, Plow E and Stenina-Adognravi O: Thrombospondin- 4 mediates TGF- $\beta$-induced angiogenesis. Oncogene 36: 5189-5198, 2017.

14. Zhang Z, Zhang X, Zhao D, Liu B, Wang B, Yu W, Li J, Yu X, Cao F, Zheng G, et al: TGF - $\beta 1$ promotes the osteoinduction of human osteoblasts via the PI3K/AKT/mTOR/S6K1 signalling pathway. Mol Med Rep 19: 3505-3518, 2019.

15. Asparuhova MB, Caballé-Serrano J, Buser D and Chappuis V: Bone-Conditioned medium contributes to initiation and progression of osteogenesis by exhibiting synergistic TGF- $\beta 1 / \mathrm{BMP}-2$ activity. Int J Oral Sci 10: 20, 2018

16. Wang $\mathrm{R}, \mathrm{Xu} \mathrm{B}$ and $\mathrm{Xu} \mathrm{H}$ : TGF- $\beta 1$ promoted chondrocyte proliferation by regulating $\mathrm{Sp} 1$ through MSC-exosomes derived miR-135b. Cell Cycle 11: 17, 2018.

17. Zhang HW, Wang EW, Li LX, Yi SH, Li LC, Xu FL, Wang DL, Wu YZ and Nian WQ: A regulatory loop involving miR-29c and Sp1 elevates the TGF- $\beta 1$ mediated epithelial-to-mesenchymal transition in lung cancer. Oncotarget 7: 85905-85916, 2016.

18. Hu J, Shan Z, Hu K, Ren F, Zhang W, Han M, Li Y, Feng K, Lei L and Feng Y: MiRNA-223 inhibits epithelial-mesenchymal transition in gastric carcinoma cells via Sp1. Int J Oncol 49: 325-335, 2016.

19. Yu S, Yerges-Armstrong LM, Chu Y, Zmuda JM and Zhang Y: Transcriptional regulation of frizzled-1 in human osteoblasts by Sp1. PLoS One 11: e0163277, 2016.

20. Duttenhoefer F, Biswas SK, Igwe JC, Sauerbier S and Bierhaus A: Sp1-Dependent regulation of PPAR $\alpha$ in bone metabolism. Int J Oral Maxillofac Implants 29: e107-e116, 2014.

21. Kim J,LeeHW,Rhee DK,Paton JC and Pyo S:Pneumolysin-induced autophagy contributes to inhibition of osteoblast differentiation through downregulation of Sp1 in human osteosarcoma cells. Biochim Biophys Acta Gen Subj 1861: 2663-2673, 2017.

22. Su F, Geng J, Li X, Qiao C, Luo L, Feng J, Dong X and Lv M: Bspl promotes tumor angiogenesis and invasion by activating VEGF expression in an acquired trastuzumabresistant ovarian cancer model. Oncol Rep 38: 2677-2684, 2017.

23. Hu H, Han T, Zhuo M, Wu LL, Yuan C, Wu L, Lei W, Jiao F and Wang LW: Elevated COX-2 expression promotes angiogenesis through EGFR/p38-MAPK/Sp1-dependent signalling in pancreatic cancer. Sci Rep 7: 470, 2017.

24. Liu M, Fan F, Shi P, Tu M, Yu C, Yu C and Du M: Lactoferrin promotes MC3T3-E1 osteoblast cells proliferation via MAPK signaling pathways. Int J Biol Macromol 107: 137-143, 2018.

25. Zhai F, Song N, Ma J, Gong W, Tian H, Li X, Jiang C and Wang H: FGF18 inhibits MC3T3E1 cell osteogenic differentiation via the ERK signaling pathway. Mol Med Rep 16: 4127-4132, 2017.

26. Livak KJ and Schmittgen TD: Analysis of relative gene expression data using real-time quantitative PCR and the 2(-Delta Delta C(T)) method. Methods 25: 402-408, 2001.

27. Chen Y, Zhang Q, Charles EB, Wu Y, Zhang L, Dong J and Han Y: VEGF overespression promotes the proliferation and differentiation of human adipose-derived stem cells. Xi Bao Yu Fen Zi Mian Yi Xue Za Zhi 33: 352-356, 2017 (In Chinese).

28. Bhattacharya R, Fan F, Wang R, Ye X, Xia L, Boulbes D and Ellis LM: Intracrine VEGF signalling mediates colorectal cancer cell migration and invasion. Br J Cancer 117: 848-855, 2017.

This work is licensed under a Creative Commons Attribution-NonCommercial-NoDerivatives 4.0 International (CC BY-NC-ND 4.0) License. 